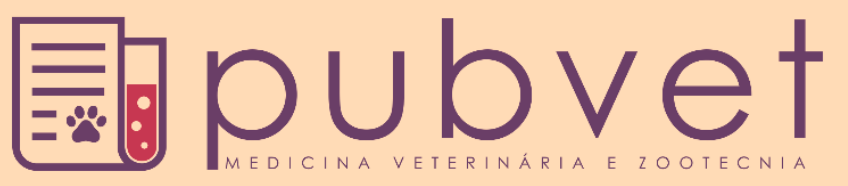

https://doi.org/10.31533/pubvet.v13n10a437.1-5

\title{
Tripanossomose bovina em um rebanho leiteiro no município de Monte Carmelo, Minas Gerais: relato de caso
}

\author{
Alexandre Lifonso de $\operatorname{Souza}^{10}$, Alexandre Luiz Alves ${ }^{1}{ }^{\ominus}$, Alexandre Resende Rocha ${ }^{1}{ }^{\theta}$, Ana \\ Vitória Ferreira Borges $^{1 \oplus}$, Kaio Batista dos $\operatorname{Reis}^{1}{ }^{\ominus}$, Lara Cristina de Souza e Silva $^{1}{ }^{\ominus}$, Lorena \\ Carolina de Oliveira $^{1}{ }^{\bullet}$, Luciana Argenton Magalhães ${ }^{1}$, Margarete Lázara de Oliveira \\ Gonçalves $^{10}$, Natalia Cristina Reis Lemes ${ }^{10}$, Tomaz dos Reis Oliveira ${ }^{1}$, Wesley Fernandes \\ Silva $^{1}$, Cláudio $\operatorname{Costa}^{2} \theta$, Francielle Aparecida de Sousa ${ }^{3} \bullet$, Laryssa Freitas Ribeiro ${ }^{2 *} \bullet$ \\ ${ }^{I}$ Alunos do curso de Medicina Veterinária do $2^{\circ}$ período do Centro Universiário Mário Palmério,, Monte Carmelo, Minas Gerais, Brasil \\ ${ }_{2}^{2}$ Professores do curso de Medicina Veterinária do Centro Universiário Mário Palmério,, Monte Carmelo, Minas Gerais, Brasil \\ ${ }^{3}$ Professora do curso de Medicina Veterinária do Centro Universitário do Cerrado, Patrocínio, Minas Gerais, Brasil. \\ *Autor de correspondência: laryssaribeiro84@gmail.com
}

Resumo. A tripanossomose bovina, cujo agente é o Trypanosoma vivax, é de grande importância atualmente, pois tem sido reportada em todo país através de surtos, acometendo principalmente bovinos leiteiros, causando grandes prejuízos aos pecuaristas. O objetivo deste estudo foi relatar o caso de tripanossomose bovina no município de Monte Carmelo MG, abordando os aspectos clínicos, patológicos, tratamento e controle da doença gerando informações para acadêmicos, profissionais e produtores rurais.

Palavras chave: bovino, bovinocultura leiteira, Trypanosoma vivax

\section{Bovine trypanosomiasis in a dairy herd in Monte Carmelo, Brazil: case report}

\begin{abstract}
Trypanosoma vivax is bovine trypanosomosis agent and it has a great importance today as it has been reported throughout the country through outbreaks, affecting mainly dairy cattle, causing great damage to ranchers. The aim of the study was reporting the case of bovine trypanosomiasis in Monte Carmelo, Brazil. Therefore, was approached the clinical and pathological aspects, treatment and disease control generating information for academics, professionals and farmers.
\end{abstract}

Keywords: Trypanosoma vivax, dairy cattle, bovine

\section{Tripanosomiasis bovina en un hato lechero del municipio de Monte Carmelo, Brasil: Reporte de caso}

Resumen. La tripanosomiasis bovina, cuyo agente es Trypanosoma vivax, hoy en día es de gran importancia, ya que se ha notificado en todo el país a través de epidemias, que afectan principalmente el ganado lechero, causando grandes daños a los ganaderos. El objetivo de este estudio fue reportar un caso epidemiológico de Tripanosomiasis bovina y sus aspectos clínicos, patológicos, de tratamiento y control de la enfermedad generando información para académicos, profesionales y productores rurales.

Palabras clave: ganado, ganadería lechera, Trypanosoma vivax 


\section{Introdução}

Tripanossomose bovina é uma doença causada por um grupo de protozoários patogênicos do gênero Trypanosoma, que tem larga distribuição e importância econômica mundial (Adam et al., 2012). No Brasil, a ocorrência por T. vivax está se tornando cada vez mais frequente em vários estados (Batista et al., 2007; Cadioli et al., 2012; Cuglovici et al., 2010; Galiza et al., 2011; Pimentel et al., 2012).

A doença apresenta diferentes situações epidemiológicas em diferentes regiões do Brasil. O Norte e o Pantanal de Mato Grosso podem ser considerados áreas de estabilidade enzoótica para a tripanossomíase, onde a doença é frequentemente benigna e os animais infectados são assintomáticos, com baixíssima morbidade e mortalidade (Silva et al., 2003). Em áreas não endêmicas, como a região semiárida do Nordeste do Brasil, os surtos ocorrem mais frequentemente durante a estação chuvosa, quando há um aumento nas populações de vetores. Porém, de forma geral, os surtos descritos em rebanhos bovinos são relatados com alta mortalidade, abortos, repetição de calor, anestro, mortes neonatais e redução drástica da produção de leite (Batista et al., 2011; Galiza et al., 2011; Rodrigues et al., 2013). Assim, os impactos econômicos causados na produção se devem ao amplo espectro de vetores e hospedeiros susceptíveis e à imunodeficiência dos animais, em sua maioria subnutrida (García et al., 2006). Além disso, o coeficiente de morbidade, o qual varia durante os surtos, pode chegar a $70 \%$, principalmente se os animais não forem tratados (Radostits et al., 2010).

O ciclo biológico do T. vivax envolve o hospedeiro mamífero e a mosca tsé-tsé pertencente ao gênero Glossina (Lopes et al., 2018). Também pode ocorrer a transmissão do modo iatrogênico, ou seja, pela utilização de uma agulha por vários animais durante a aplicação de medicamentos ou vacinações (Jones \& Dávila, 2001; Vargas \& Arellano, 1997).

Sabendo dos prejuízos econômicos que a doença pode causar e a facilidade de transmissão do agente, o objetivo do estudo foi fazer uma revisão abordando os aspectos clínicos, patológicos, as técnicas de diagnóstico, tratamento e controle da doença gerando informações para acadêmicos, profissionais e produtores rurais, auxiliando, assim, para uma diminuição dos casos e consequente, diminuição dos prejuízos causados.

\section{Relato de caso}

Este relato se pauta em um estudo de caso realizado em uma fazenda de produção leiteira no município de Monte Carmelo, Minas Gerais, no mês de março de 2019. O rebanho era constituído por 130 animais, com 105 vacas em lactação, com uma produção de 1.600 litros de leite por dia. O manejo da ordenha era realizado duas vezes ao dia, com o auxílio do hormônio ocitocina, o qual era aplicado antes da retirada do leite, utilizando-se a mesma seringa em todos os animais.

A demanda do caso em questão fora apresentada pelo dono, responsável pela produção leiteira da propriedade que, no contato com um médico veterinário da região, relatou que quatro animais haviam morrido e um estava deitado. Os sinais clínicos apresentados foram dificuldade de locomoção, corpo duro, diarreia, perda de apetite, a perda acentuada de peso e queda na produção de leite, a qual caiu para 950L/dia.

Ademais, na visita à propriedade, observou-se um manejo de péssima higiene, porcos soltos na sala de ordenha e o uso inadequado da mesma seringa de ocitocina para todos os animais em produção. Ainda, o proprietário relatou ter comprado animais de outros municípios. $\mathrm{Na}$ anamnese, de cinco animais em estado grave, observou-se perda de apetite, baixo escore corporal, queda na produção de leite, mucosas hipocoradas e diarreia fétida e contínua.

Após a avaliação e exame físico realizado nesses animais pressupôs-se algum parasita sanguíneo, devido à mucosa pálida, e a partir do quadro em que os animais se encontravam, teve como primeira suspeita tripanossomose. $\mathrm{O}$ veterinário contatado realizou necropsia de um animal que, segundo o proprietário, apresentava sinais clínicos semelhantes aos animais avaliados. Nesta, encontrou-se esplenomegalia, hepatomegalia, mucosas hipocoradas e pontos de necrose nos dois órgãos (Figura 1).

Realizou-se, então, a coleta de sangue de três animais com sinais clínicos e as amostras foram enviadas a um laboratório especializado. O diagnóstico foi realizado a partir de um teste parasitológico direto para identificar algum hemoparasita sanguíneo. O laudo, portanto, confirmou a presença de Tripanossoma spp degenerados e íntegros, confirmando a suspeita inicial. Além disso, foi identificada 
a presença de hemácias crenadas, o que indica um quadro de desidratação e a presença de linfócitos apáticos, indicativo de leucemia.

O tratamento do rebanho foi realizado com a administração de diaceturato de 4,4 diazoamino dibenzamidina 7\% (Ganaseg plus ${ }^{\circledR}$ Novartis) por doze dias e também Vivedium, na dose de $1 \mathrm{~mL} / 20 \mathrm{~kg}$. Foi recomendada também a correta realização de manejos das seringas, para aplicação de ocitocina, o qual se indicou usar uma seringa por animal e logo após a primeira ordenha elas são colocadas de molho em um balde de água com CB 30 em alta concentração até a próxima ordenha.

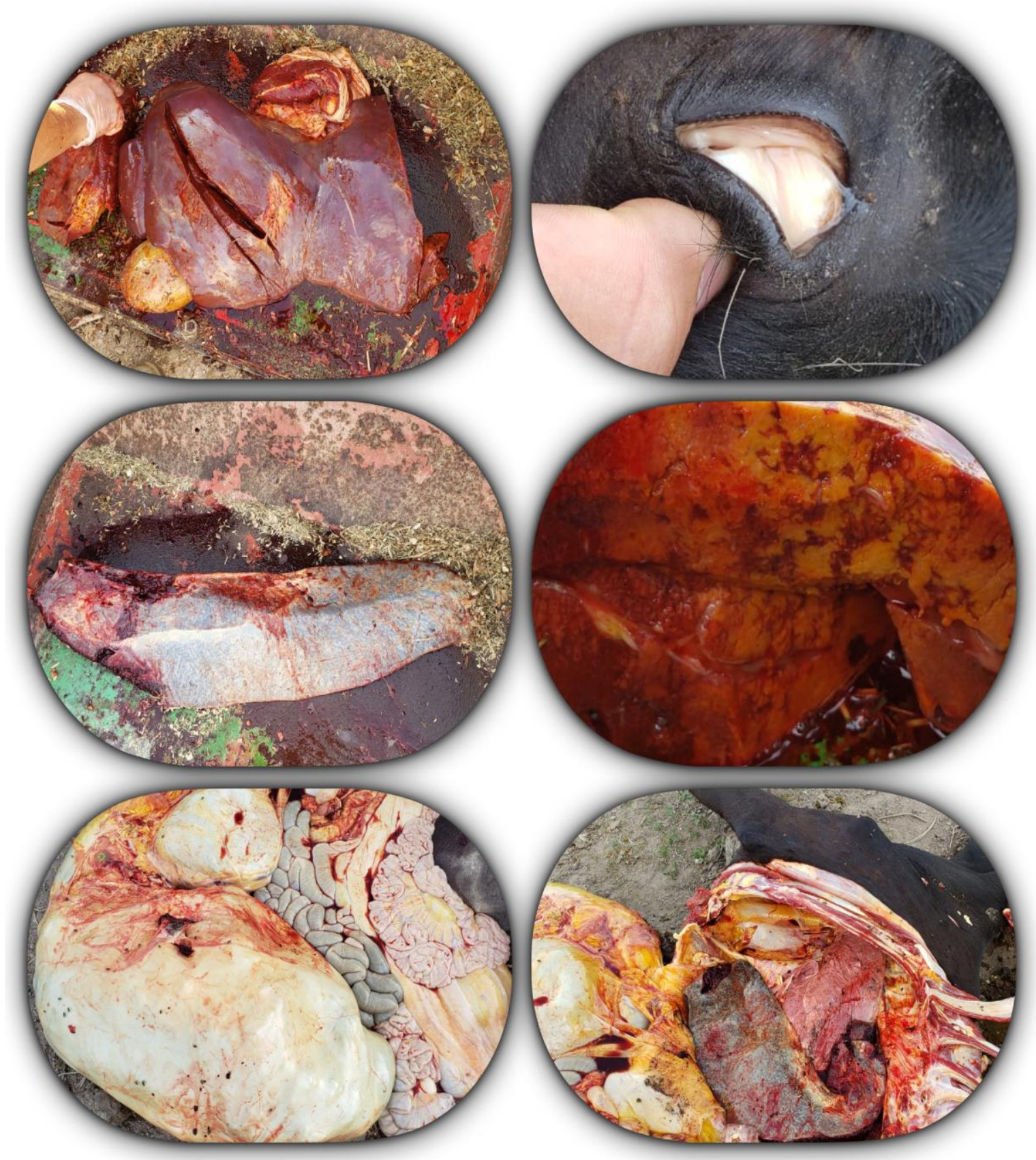

Figura 1. Achados de necropsia de um dos animais com suspeita de tripanossomose, em uma propriedade no município de Monte Carmelo, Minas Gerais. Observa-se esplenomegalia, hepatomegalia, mucosas e órgãos hipocorados e pontos de necrose nos dois órgãos.

Após o tratamento, quase todos os animais se recuperaram, com óbito de apenas três, os quais já se encontravam em fase terminal. Observou-se, posteriormente, que os sinais clínicos haviam cessado e a produção leiteira na fazenda voltou a crescer. No total, 27 animais vieram a óbito (sendo um 
necropsiado) desde o início do surto e 48 animais do total do rebanho apresentaram apenas sinais clínicos, se recuperando após o tratamento.

\section{Discussão}

No surto relatado, os animais apresentaram inapetência, perda de peso acentuada, desnutrição, queda na produção de leite, diarreia fétida e aquosa e mucosas hipocoradas. Além disso, os animais deitaramse no decorrer do quadro em decúbito esternal e permaneceram assim até o óbito. Sinais e sintomas muito parecidos também foram relatados por Batista et al. (2008). Ademais, os achados de necropsia como hepatomegalia, esplenomegalia e pontos de necrose nos dois órgãos também se assemelham aos descritos por Batista et al. (2008).

Os sinais clínicos registrados no histórico deste caso e a busca pelo hemoparasita via exame e laudo laboratorial apontou para um surto de Tripanossomose bovina, demonstrando alta parasitemia. Esta confirmação é a primeira no município de Monte Carmelo, Minas Gerais, apontando para uma preocupação regional quanto à doença que se estende em âmbito nacional; preocupação também apresentada pelos pesquisadores que estudam a Tripanossomose (Cadioli et al., 2012). Entretanto, pela literatura depreende-se que podem existir animais assintomáticos, o que para os autores é preocupante, pois prejudica programas de controle que são dependentes da manifestação clínica. Ressalta-se, então, que os assintomáticos também são relevantes como fonte de infecção e, portanto, disseminadores da doença (Berthier et al., 2016).

Na propriedade aplicava-se ocitocina duas vezes ao dia, com a mesma agulha e seringa em todos os animais do rebanho, o que pode ter favorecido a transmissão do T. vivax entre os animais, como já descrito por Vargas \& Arellano (1997) e Jones \& Dávila (2001), embora tenha sido observada a presença de moscas hematófagas. Para evitar a doença, além de controlar o vetor, é imprescindível evitar o uso compartilhado de agulhas (Germano et al., 2018).

Esse estudo de caso mostra um pouco da dimensão desse problema, que acarreta tantas perdas econômicas ao produtor rural. Perdas descritas por Abrão et al. (2009); no qual o tratamento saiu em torno de $\mathrm{R} \$ 20,00$, sem contar os prejuízos causados pela morte dos animais.

O T. vivax está presente na região (Triângulo Mineiro - Alto Paranaíba) e muitas vezes pode ter sido negligenciadas um correto diagnóstico. Germano et al. (2018) também compartilha da mesma opinião: "A falta de um diagnóstico, o desconhecimento por parte dos produtores e profissionais é um agravo para a ocorrência".

De forma geral, o controle é baseado em evitar moscas e no não compartilhamento de agulhas. Todavia, ainda são necessários mais estudos sobre a epidemiologia da doença para que ocorra um maior controle. A partir de informações, como prevalência do agente em animais domésticos e silvestres no Brasil, será possível fortalecer a proposta de um programa para orientar criadores sobre boas práticas de manejo dos animais, assim como uma maior fiscalização da doença pelas agências de defesa sanitária, impedindo, assim, a disseminação desses agentes patogênicos nas propriedades (Lopes et al., 2018).

\section{Conclusão}

O histórico dos animais, os achados de necropsia e os resultados dos exames clínicos e laboratoriais revelaram a ocorrência de um surto de tripanossomíase por T. vivax em bovinos de uma propriedade da cidade de Monte Carmelo, Minas Gerais, sendo este o primeiro registro da doença na região.

\section{Referências bibliográficas}

Abrão, D. C., Carvalho, A. Ú., Facury Filho, E. J., Saturnino, H. M. \& Ribeiro, M. F. B. (2009). Impacto econômico causado por Trypanosoma vivax em rebanho bovino leiteiro no estado de Minas Gerais. Ciência Animal Brasileira, 1(Supl.):672-676.

Adam, Y., Marcotty, T., Cecchi, G., Mahama, C. I., Solano, P., Bengaly, Z. \& Van den Bossche, P. (2012). Bovine trypanosomosis in the upper west region of Ghana: Entomological, parasitological and serological cross-sectional surveys. Research in Veterinary Science, 92(3):462-468. 
Batista, J. S., Bezerra, F. S. B., Lira, R. A., Carvalho, J. R. G., Rosado Neto, A. M., Petri, A. A. \& Teixeira, M. M. G. (2008). Aspectos clínicos, epidemiológicos e patológicos da infecção natural em bovinos por Trypanosoma vivax na Paraíba. Pesquisa Veterinária Brasileira, 28(1):63-69.

Batista, J. S., Riet-Correa, F., Teixeira, M. M. G., Madruga, C. R., Simões, S. D. V. \& Maia, T. F. (2007). Trypanosomiasis by Trypanosoma vivax in cattle in the Brazilian semiarid: Description of an outbreak and lesions in the nervous system. Veterinary Parasitology, 143(2):174-181.

Batista, J. S., Rodrigues, C. M. F., García, H. A., Bezerra, F. S. B., Olinda, R. G., Teixeira, M. M. G. \& Soto-Blanco, B. (2011). Association of Trypanosoma vivax in extracellular sites with central nervous system lesions and changes in cerebrospinal fluid in experimentally infected goats. Veterinary Research, 42(1):63.

Berthier, D., Brenière, S. F., Bras-Gonçalves, R., Lemesre, J.-L., Jamonneau, V., Solano, P., . . . Bucheton, B. (2016). Tolerance to trypanosomatids: a threat, or a key for disease elimination? Trends in Parasitology, 32(2):157-168.

Cadioli, F. A., Barnabé, P. d. A., Machado, R. Z., Teixeira, M. C. A., André, M. R., Sampaio, P. H., . . . Marques, L. C. (2012). First report of Trypanosoma vivax outbreak in dairy cattle in São Paulo state, Brazil. Revista Brasileira de Parasitologia Veterinária, 21(2):118-124.

Cuglovici, D. A., Bartholomeu, D. C., Reis-Cunha, J. L., Carvalho, A. U. \& Ribeiro, M. F. B. (2010). Epidemiologic aspects of an outbreak of Trypanosoma vivax in a dairy cattle herd in Minas Gerais state, Brazil. Veterinary Parasitology, 169(3-4):320-326.

Galiza, G. J. N., Garcia, H. A., Assis, A. C. O., Oliveira, D. M., Pimentel, L. A., Dantas, A. F. M., . . . Riet-Correa, F. (2011). High mortality and lesions of the central nervous system in trypanosomosis by Trypanosoma vivax in Brazilian hair sheep. Veterinary Parasitology, 182(2-4):359-363.

García, H., García, M. E., Pérez, G., Bethencourt, A., Zerpa, E., Pérez, H. \& Mendoza-León, A. (2006). Trypanosomiasis in Venezuelan water buffaloes: association of packed-cell volumes with seroprevalence and current trypanosome infection. Annals of Tropical Medicine \& Parasitology, 100(4):297-305.

Germano, P. H. V., Silva, A. A., Edler, G. E. C., Lopes, M. C., Modesto, T. C. \& Reis, J. A. (2018). Tripanossomose bovina: revisão. PUBVET, 12( a144):1-6.

Jones, T. W. \& Dávila, A. M. R. (2001). Trypanosoma vivax-out of Africa. Trends in Parasitology, 17(2):99-101.

Lopes, S. T. P., Prado, B. S., Martins, G. H. C., Beserra, H. E. A., Souza Filho, M. A. C., Evangelista, L. S. M. \& Souza, J. A. T. (2018). Trypanosoma vivax in dairy cattle. Acta Scientiae Veterinariae, 4615

Pimentel, D. S., Ramos, C. A. N., Ramos, R. A. N., Araújo, F. R., Borba, M. L., Faustino, M. A. G. \& Alves, L. C. (2012). First report and molecular characterization of Trypanosoma vivax in cattle from state of Pernambuco, Brazil. Veterinary Parasitology, 185(2-4):286-289.

Radostits, O. M., Gay, C. C., Blood, D. C., Hinchcliff, K. W. \& McKenzie, R. A. (2010). Clínica Veterinária: um tratado de doenças dos bovinos, ovinos, suínos, caprinos e eqüinos (Vol. 1). Rio de Janeiro: Guanabara Koogan.

Rodrigues, C. M. F., Olinda, R. G., Silva, T. M. F., Vale, R. G., da Silva, A. E., Lima, G. L. \& Batista, J. S. (2013). Follicular degeneration in the ovaries of goats experimentally infected with Trypanosoma vivax from the Brazilian semi-arid region. Veterinary Parasitology, 191(1-2):146-153.

Silva, R., Rivera D'Ávila, A. M., Seidl, A. \& Ramirez, L. (2003). Trypanosoma evansi e trypanosoma vivax: biologia, diagnóstico e controle. Embrapa Pantanal, 141.

Vargas, T. M. \& Arellano, S. C. (1997). La tripanosomiasis bovina en América Latina y el Caribe. Vet Montividel, 3317-21.

Recebido: 21 de agosto, 2019.

Aprovado: 5 de novembro, 2019.

Publicado: 3 de dezembro, 2019.

Licenciamento: Este artigo é publicado na modalidade Acesso Aberto sob a licença Creative Commons Atribuição 4.0 (CC-BY 4.0), a qual permite uso irrestrito, distribuição, reprodução em qualquer meio, desde que o autor e a fonte sejam devidamente creditados. 